\title{
Lung mesenchymal expression of Sox9 plays a critical role in tracheal development
}

\author{
Gianluca Turcatel ${ }^{1}$, Nicole Rubin ${ }^{1}$, Douglas B Menke², Gary Martin ${ }^{3}$, Wei Shi ${ }^{1}$ and David Warburton ${ }^{1 *}$
}

\begin{abstract}
Background: Embryonic lung development is instructed by crosstalk between mesenchyme and epithelia, which results in activation of transcriptional factors, such as Sox9, in a temporospatial manner. Sox9 is expressed in both distal lung epithelium and proximal lung mesenchyme. Here, we investigated the effect of lung mesenchymespecific inducible deletion of Sox9 during murine lung development.

Results: Transgenic mice lacking Sox9 expression were unable to breathe and died at birth, with noticeable tracheal defects. Cartilage rings were missing, and the tracheal lumen was collapsed in the mutant trachea. In situ hybridization showed an altered expression pattern of Tbx4, Tbx5 and Fgf10 genes and marked reduction of Collagen2 expression in the tracheal mesenchyme. The tracheal phenotype was increasingly severe, with longer duration of deletion. Lymphatic vasculature was underdeveloped in the mutant trachea: Prox1, Lyve1, and Vegfr3 were decreased after Sox9 knockout. We also found that compared with normal tracheal epithelium, the mutant tracheal epithelium had an altered morphology with fewer P63-positive cells and more CC10-positive cells, fewer goblet cells, and downregulation of surfactant proteins $A$ and $C$.

Conclusion: The appropriate temporospatial expression of Sox9 in lung mesenchyme is necessary for appropriate tracheal cartilage formation, lymphatic vasculature system development, and epithelial differentiation. We uncovered a novel mechanism of lung epithelium differentiation: tracheal cartilage rings instruct the tracheal epithelium to differentiate properly during embryonic development. Thus, besides having a mechanical function, tracheal cartilage also appears to be a local signaling structure in the embryonic lung.
\end{abstract}

Keywords: Sox9, Trachea, Lung, Cartilage, CC10, P63

\section{Background}

The development of the respiratory system represents an evolutionary hallmark that allowed vertebrates to survive on land utilizing air as a source of oxygen. Abnormal development of the respiratory system in humans is associated with multiple disorders such as tracheal/bronchial atresia, tracheo-esophageal fistula, bronchogenic cysts, pulmonary/lobar atresia, and pulmonary hypoplasia [1].

In the embryonic trachea, the endoderm differentiates into a ciliated pseudo-stratified epithelium, which includes basal P63+ cells, Clara cells, neuroendocrine cells, and ciliated cells. The ventral mesenchyme gradually matures

\footnotetext{
* Correspondence: dwarburton@chla.usc.edu

'Developmental Biology and Regenerative Medicine Program, Saban

Research Institute, Children's Hospital Los Angeles, Keck School of Medicine and Ostrow School of Dentistry, University of Southern California, 4661

Sunset Boulevard, Los Angeles, CA 90027, USA

Full list of author information is available at the end of the article
}

to create $\mathrm{C}$-shaped cartilage rings, while the dorsal mesenchyme forms contractile cells comprising the trachealis smooth muscle, which defines the posterior boundary between the trachea and esophagus. Tracheal wall malformation can be due to congenital or acquired abnormalities. The congenital forms are related to immature tracheal cartilage, whereas the more common acquired forms occur as a consequence of compression damage or degeneration of the tracheal cartilage, such as in vascular sling anomalies [2]. Tracheomalacia is a condition described as causing excessive expiratory collapse of the trachea, resulting from weakness of the supporting structures of tracheal and bronchial walls, leading to symptoms of airway obstruction $[2,3]$.

$\beta$-catenin, Bmp4, and Shh are previously well-characterized regulators of cartilage formation, which may activate the Sox9 gene in mesenchymal cells to induce their differentiation into chondrocytes. Members of the fibroblast growth 
factor superfamily, including $F g f 4, F g f 8$, and $F g f 10$, are also key regulators of cartilage formation [4,5]. Fgf18 regulates proliferation and spatial localization of the chondrocytes in the developing upper respiratory tract [6]. Tbx4 and $T b \times 5$ double knockout in lung resulted in defects of cartilage formation in mouse trachea and in altered lung branching, with $T b x 4$ and $T b x 5$ acting upstream of Sox9 in tracheal cartilage development [7].

Here, we used an inducible Tbx4 gene-driven system to knock out the Sox9 gene specifically in lung mesenchyme [8], in order to elucidate more fully its function within the mesenchyme during lung development. Mesenchymal Sox9 knockout had no affect on distal lung branching, on formation of smooth muscle around the bronchi, or on the trachealis smooth muscle, whereas tracheal cartilage formation was completely inhibited. In situ hybridization revealed that in the Sox9 null trachea, the characteristic Tbx4, Tbx5, and Fgf10 expression pattern was lost or severely disrupted.

Mutant Sox9 mouse tracheal epithelium was also altered: cells had a higher number of cytoplasmic vacuoles, with fewer basal cells (P63+ cells) and more Clara cells (CC10+ cells). Mutant tracheal epithelium also had fewer goblet cells compared with wild-type trachea. Surfactant proteins A and $\mathrm{C}$ were also downregulated. Finally, tracheal lymphatic vessels were significantly underdeveloped after the knockout of mesenchymal Sox9. In conclusion, mesenchymal Sox9-expressing cells cover a critical developmental role in the formation of tracheal cartilage rings, and in the appropriate differentiation of lung tracheal epithelium and lymphatic system.

\section{Results}

\section{Sox9 expression during lung development}

We used western blot to analyze the expression of Sox9 in mouse lung during development. Sox9 protein expression level is high in embryonic lungs but drastically decreases in mouse lung after birth. Adult mouse lung does not express Sox9 protein at all (Figure 1A). At embryonic day (E)12.5, Sox9 is expressed by distal lung epithelial cells and by a population of mesenchymal cells surrounding the trachea, bronchi, and bronchioles. At E15.5, mesenchymal cells expressing Sox9 cells are condensed at the area of the future cartilage rings, while epithelial cells expressing Sox9 are localized in the distal lung epithelium (Figure 1B). To specifically knock out Sox9 in mesenchymal cells, we used a Tbx4-rtTA/Tet-On-Cre system, in which rtTA gene expression is regulated by the mesenchymal specific enhancer of the $T b x 4$ gene $[8,9]$. Tbx4 is expressed by tracheal mesenchyme and most of the lung distal mesenchyme (Figure 1C). Sox9 is efficiently deleted in the Tbx4-rtTA/Tet-On-Cre/Sox $9^{\mathrm{fl} / \mathrm{fl}}$ at E12.5 and E15.5 stage (Figure 1D-I).

\section{Mesenchymal Sox9 knockout mice showed an absence of tracheal cartilage rings}

Perl reported that epithelial Sox9 deletion did not affect lung development [10]. However, more recently, Chang showed that Sox9 deletion in lung epithelium resulted in a smaller lung, with fewer and dilated airway branches [11]. It is possible that the use of different mouse genetic backgrounds is the cause of these contrasting results. The role of mesenchymal Sox 9 expression is unknown. In our study, transgenic mice lacking mesenchymal Sox 9 expression were born at the expected mendelian frequency and appeared normal at birth, but rapidly became cyanotic and showed marked signs of respiratory obstruction including gasping and retractions (Figure 2A); moreover, not a single transgenic mouse lived more than a few hours after birth (Figure 2B). Despite the lethal respiratory obstruction phenotype, no difference in weight at birth was observed (Figure 2C). At both E15.5 and E18.5, embryonic lung branching did not appear to be affected by lack of mesenchymal Sox9 expression (Figure 2D,E; see Additional file 1: Figure S1A-D). However, transgenic embryos were missing cartilage rings around the trachea and bronchi. (Figure 2F-I; see Additional file 1: Figure S1E,F). Transverse sections of transgenic embryo E18.5 tracheas showed collapsed airway lumen, and a shape and appearance similar to esophagus; however, the esophagus was still present. In $8 \%$ of the embryos, rudimentary cartilage spots developed on the ventral side of the trachea (see Additional file 2: Figure S2B black arrowheads). These spots were still expressing Sox9 protein, indicating the existence of another unique population of Sox9+ cells that either did not continue to express the Tbx4 gene or did not express the transgene, or expressed the transgene, but did not recombine (see Additional file 2: Figure S2C). Lung branching was not affected by lack of mesenchyme Sox9 expression (Figure 3A-C), and differentiation of distal lung epithelium and mesenchyme appeared to be normal in the mutants (Figure 3D-G).

\section{The tracheal phenotype depends on the timing of doxycycline exposure}

The observed phenotype (Figure 2; see Additional file 1: Figure S1) was obtained by knocking out the Sox9 gene in the mesenchyme of the lung, starting from day E7.5 to the day of collection of the embryos (E18.5). We then exposed pregnant time-mated females to different durations of doxycycline induction to determine the relation between phenotype and doxycycline induction (Figure 4A). Shorter exposure to doxycycline resulted in a milder phenotype (Figure 4B-G), as embryos exposed to doxycycline for shorter time periods developed some of the more distal cartilage rings. If doxycycline was removed from the food at E11.5, the two most proximal cartilage rings were fully formed (Figure 4F,G, black arrows). Sox 9 

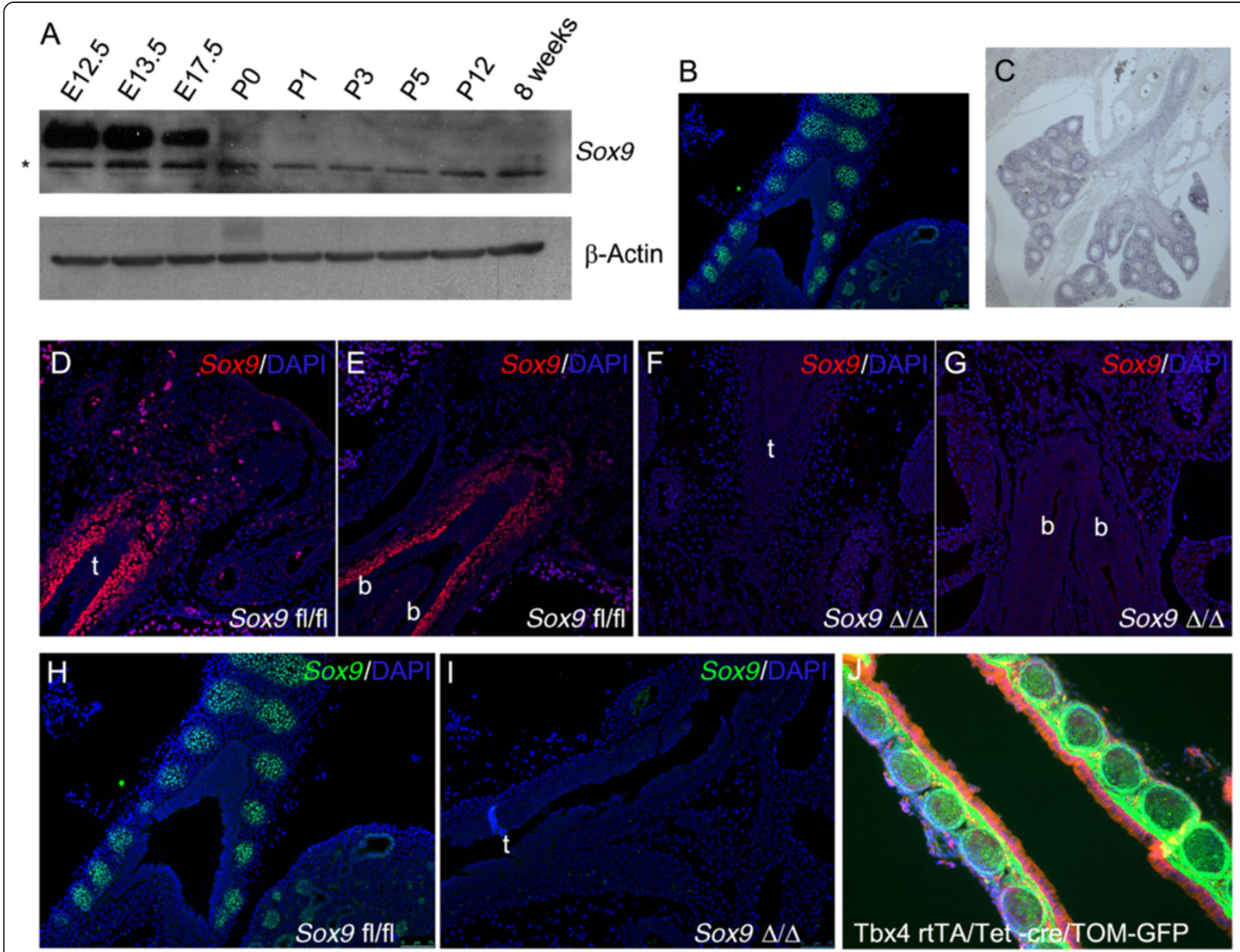

Figure 1 Expression of Sox9 during lung development. (A) Western blot analysis of Sox9 protein expression during lung development at different stages (*non-specific bands). (B) Sox9 protein expression at E15.5 stage: immunofluorescence localization of Sox9 showed expression of Sox9 by tracheal immature cartilage and distal lung epithelium. (C) Expression pattern of endogenous Tbx4 gene in E15.5 lung: Tbx4 is expressed by tracheal mesenchyme and by most, but not all of the distal lung mesenchyme. (D-I) Sox9 immunostaining, showing effective deletion of mesenchyme Sox-9 expression in the mutant lungs at embryonic day (D-G) E12.5 and (H, I) E15.5 stage. (J) Cell tracing using Tbx4-rtTA line. Tbx4-rtTA/Tet-on-cre males were bred with $\mathrm{mT} / \mathrm{mG}$ females. E7.5 staged pregnant females were fed with doxocycline, and E18.5 embryos were collected. Lung epithelial cells expressed red fluorescent protein, while the mesenchyme cells are green because they are derived from Tbx4-expressing cells (t, trachea; b, bronchus).

knockout in E14.5 embryos did not induce any abnormalities in the cartilage development, as Tbx4 and Sox9 protein are not co-expressed in the tracheal mesenchyme at that stage (Figure $4 \mathrm{H}-\mathrm{J}$ ).

\section{Expression pattern of Tbx4, Tbx5, and Fgf10 is altered in Sox9 mutant trachea}

We next determined whether the expression pattern of genes known to be involved in tracheal cartilage ring formation was altered in the mutant trachea. Tbx4 and Tbx 5 are expressed between the tracheal cartilage rings in normal mouse trachea [6]. In situ hybridization on E15.5 embryonic lung tissue revealed that $T b x 4$ and Tbx5 expression patterning was lost in the mutant trachea (Figure 5A-D). Tbx4 and Tbx5 were expressed uniformly by all the mesenchyme lining the trachea (Figure 5B,D), instead of there being an alternating striped pattern as observed in the control trachea (Figure 5A,C). Using real-time reverse transcription quantitative PCR (RT-qPCR) on RNA extracted from E15.5 tracheas, we observed increased expression of $T b \times 4$ and $T b \times 5$ in the mutant trachea versus the control trachea (Figure 5G). Fgf10 is another known key regulator of tracheal cartilage development. The Fgf10 expression pattern in the E15.5 stage trachea normally delimits the primitive cartilage rings; however, this specific expression pattern was lost after Sox9 knockout, and Fgf10 was expressed by all the tracheal mesenchyme 

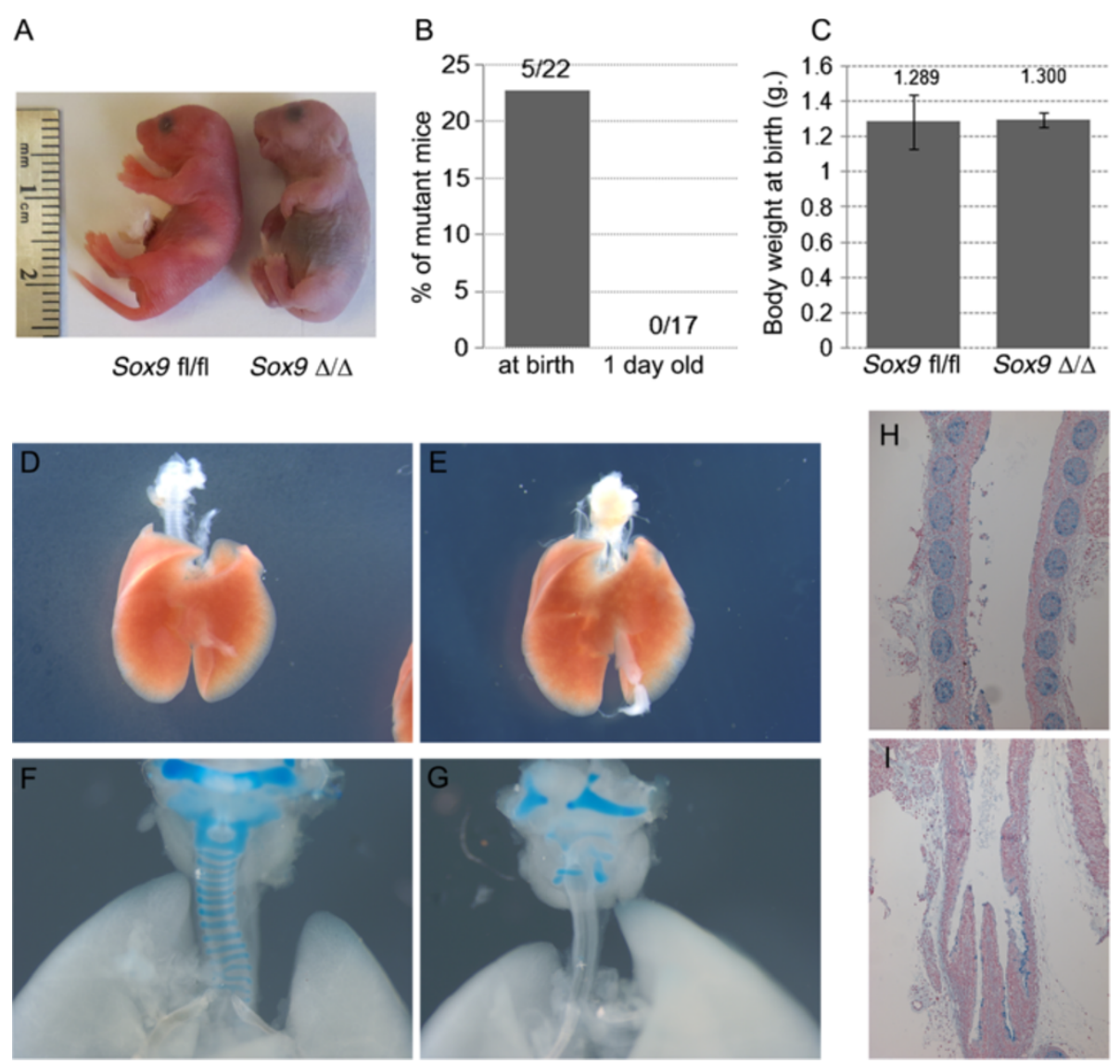

Figure 2 Lung phenotype after mesenchymal Sox 9 knockout. (A) Pups were collected at birth. Mutant pups died less than an hour after birth, and showed gasping, retractions, and cyanosis. (B) Mutant pups did not survive post-natally. (C) Despite the respiratory mortality phenotype, no change in weight at birth was observed between the wild-type pups and the mutant pups. (D-I) Samples of lung at embryonic day (E) 18.5 were collected and analyzed. (E) Sox $9^{\Delta / \Delta}$ did not show obvious altered lung branching compared with (D) the control. (F, G) Alcian blue staining of (F) E18.5 wild-type and (G) mutant lungs: normal tracheal rings were observed in the control normal lung, Sox9 knockout trachea revealed no tracheal rings. $(\mathbf{H}, \mathbf{I})$ Transverse section of E18.5 trachea was stained with Alcian blue. (H) Wild-type and (I) mutant mice.

(Figure 5E,F). We observed a trend towards decreased Fgf10 mRNA expression in the mutant trachea but it was not statistically significant.

\section{Trachealis contractile muscle pattern formation and bronchial smooth muscle cells are not affected by Sox 9 knockout}

The Tbx4, Tbx5, and Fgf10 expression patterns were lost in Sox9 mutants. This observation led us to investigate whether the Sox9 gene is also necessary for patterning the SM-22- $\alpha+$ cells, which are located at the junction between the trachea and esophagus (dorsal trachea), and form the trachealis muscle. Because Sox9-expressing cells are located at the ventral region of the trachea, $S m-22-\alpha$ and Sox9 expression seems to be mutually exclusive, suggesting that expression of Sox9 is limiting the expression of Sm-22- $\alpha$ to the dorsal area of the trachea, and vice versa.

However, we found that Sox 9 expression is not involved in patterning the expression of $S m-22-\alpha$-expressing cells in the trachea (Figure 5F-K). In fact, the contractile lunarshaped ring of Sm-22- $\alpha+$ cells was still present and localized in the dorsal area of the mutant trachea. The absolute area covered by Sm22- $\alpha+$ cells did not change significantly in the mutant trachea versus the wild-type trachea (Figure 5J). We also analyzed whether the Sox 9 gene was involved in the proliferation of bronchial smooth muscle cells; however, we did not observe any obvious change in smooth muscle cells lining the bronchi of mutant mouse lung (Figure 5L,M).

\section{Tracheal epithelium is altered after Sox9 knockout}

Mice lacking Sox9 in the lung mesenchyme died at birth, due to an inability to breathe because of the collapsed trachea. No change in smooth muscle cell numbers or patterning was observed. However, the epithelium lining the trachea was abnormal; epithelial cells in the mutant trachea had larger and more numerous vacuoles (Figure 6B,F). Electron microscopy revealed that some of those cells 


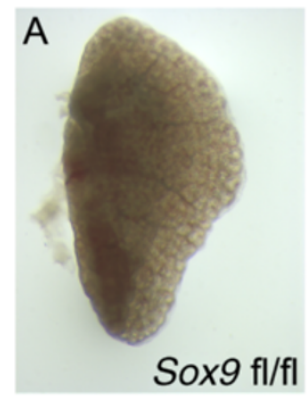

B
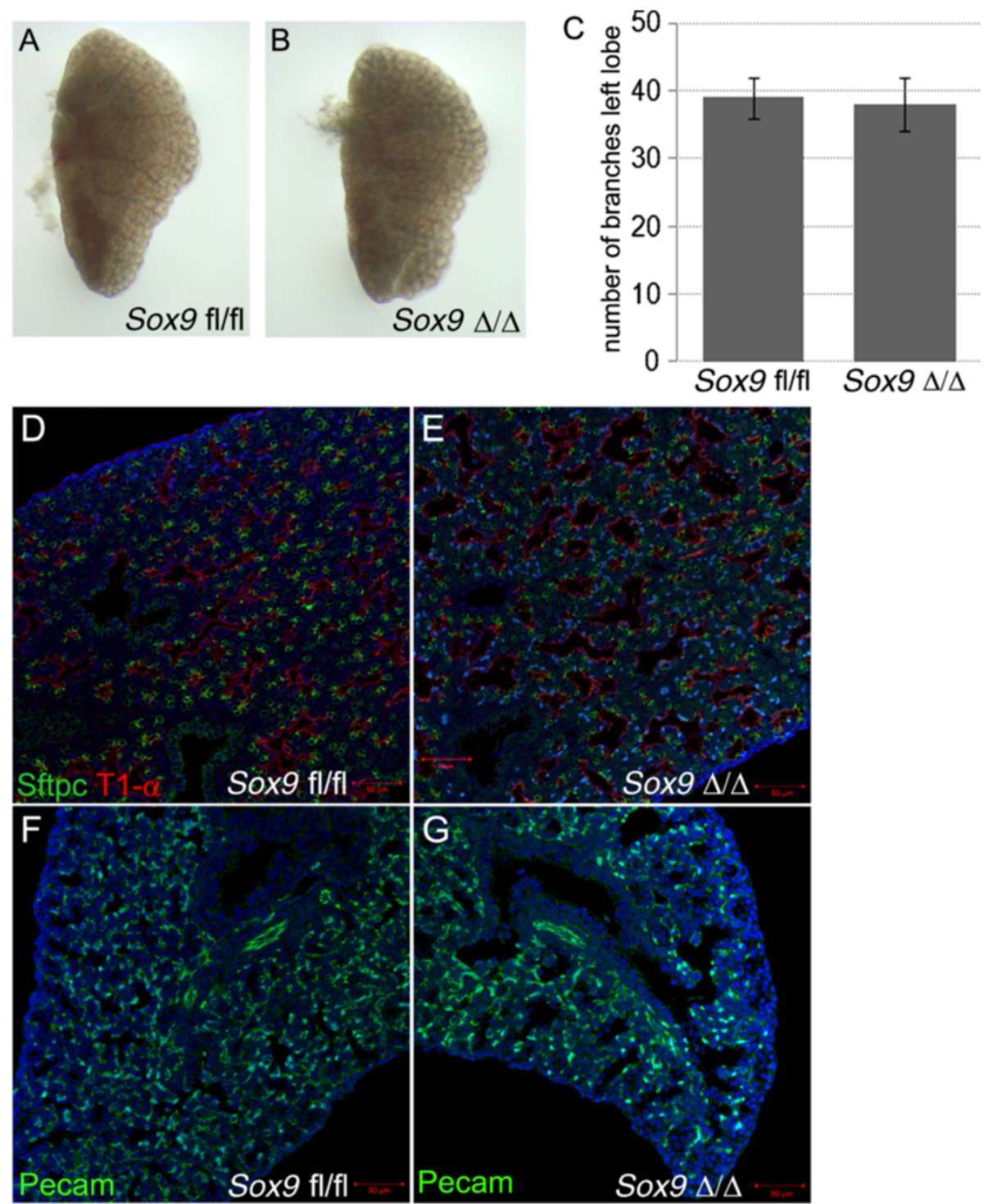

Figure 3 Mesenchymal Sox9 deletion did not alter lung branching. (A, C) Left lobes of (A) wild-type and (B) mutant lung at embryonic day (E) 15.5. Terminal branches were counted, and no difference in branching morphogenesis was identified. (D-G) Immunostaining for Sftpc, T1-ac, and Pecam in E18.5 lungs confirmed that distal lung epithelium and mesenchyme differentiation were not altered by Sox9 deletion in the lung mesenchyme.

looked like Clara cells (Figure 6G). Real-time RT-qPCR was used to quantify change of expression of lungrelevant genes in mutant mouse trachea. Expression of the surfactant protein genes $S f t p-a$ and $S f t p-c$ mRNA was reduced in the $\operatorname{Sox}^{\Delta / \Delta}$ mice, and CC10 mRNA was slightly overexpressed in the mutant trachea (Figure $6 \mathrm{H}$ ), indicating altered differentiation of tracheal epithelium. The number of ciliated cells did not change after mesenchymal deletion of the Sox9 gene (Figure 7A,B,G). However, at E18.5, the number of tracheal basal cells (P63+) decreased (Figure 7C,D,H) and Clara cells (CC10+) (Figure 7E,F,I) increased in the mutant trachea versus the control trachea. E15.5 $\operatorname{Sox}^{f l / f l}$ and $\operatorname{Sox} 9^{\Delta / \Delta}$ tracheas showed a similar number of P63+ cells (see Additional file 3: Figure S3). Alcian blue staining of transverse sections of trachea revealed decreased mucin production by the mutant tracheal epithelium (Figure 6E,F). Agr2 protein, a marker of lung goblet cells, was downregulated in the tracheal epithelium of the $\operatorname{Sox} 9^{\Delta / \Delta}$ embryos compared with the $\operatorname{Sox} 9^{f l / f l}$ embryos (Figure 7J-M). However, expression of Sox2 and Foxp1, key regulators of lung epithelium differentiation, was unaltered after Sox9 knock out (see Additional file 4: Figure S4).

Despite the altered differentiation, proliferation of tracheal epithelium was not affected by lack of mesenchymal Sox9 expression, No change in phospho-histone-3 


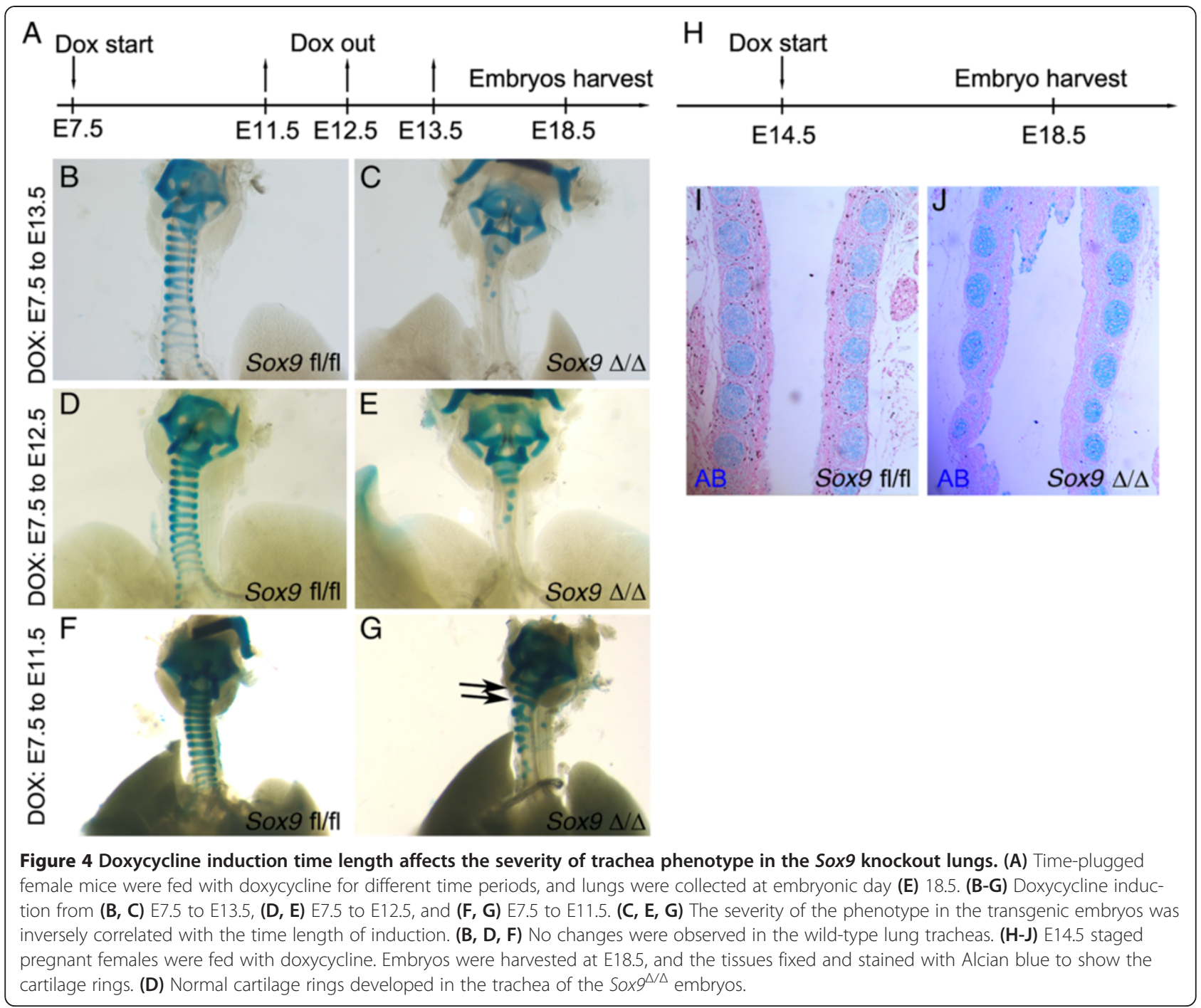

(pH3) and total cell number per tracheal transversal section was found (Figure 8A-E).

\section{Tracheal lymphatic system was altered in Sox $9^{\Delta / \Delta}$ trachea}

We quantified the area covered by lung mesenchyme (excluding the cartilage) and the mesenchyme cell number on transverse sections of E18.5 wild-type and mutant tracheas. Despite the somewhat misleading visual appearance, both the mesenchyme cell numbers and the surface covered by them were the same in the Sox $9^{f l / f l}$ and So $x 9^{\Delta / \Delta}$ trachea (Figure 9A-C,C'). Prox1 and Lyve1 are key markers of lymphatic vessel development [12]. Prox 1 also controls neuronal progenitor cell differentiation to maintain the required balance between different cell types [13]. We found that the tracheal lymphatic system was significantly underdeveloped in the mutant Sox9 embryos, as shown by reduced immunostaining of Prox1, Lyve1, and Pecam (Figure 9F-K). In addition, the,
mRNA level of Prox1, Lyve1, and Vegfr3 was reduced in the $\operatorname{Sox} 9^{\Delta / \Delta}$ tracheas (Figure 9l).

\section{Discussion}

Normal lung branching is achieved by tightly regulated crosstalk between mesenchymal and epithelial compartments. Specific growth factors are released from one compartment to activate expression of genes in the other compartment, and vice versa $[14,15]$. Sox9 is a multifunctional transcriptional factor expressed in both embryonic lung mesenchyme and epithelium. Perl et al. reported that deletion of Sox9 gene in embryonic lung epithelium did not affect lung development; mice lacking Sox9 in the lung epithelium were viable and fertile, and did not show any differences from their control littermate, even when exposed to hyperoxia-induced injury [10]. However, more recently, Chang et al. showed that Sox9 is necessary for proper lung branching [11]. Sox9 

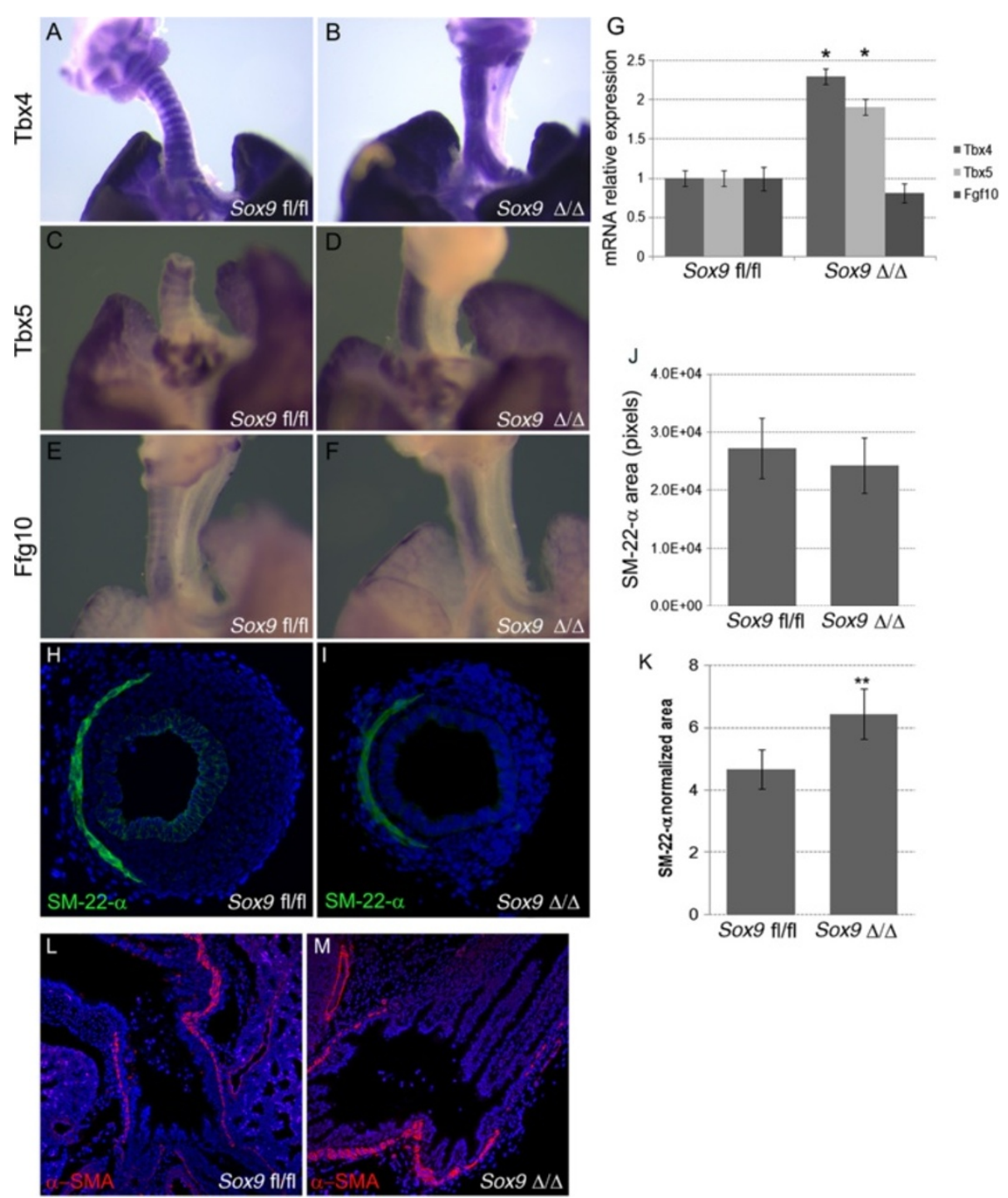

Figure 5 Tbx4, Tbx5, and Fgf10 in situ hybridization results. In situ hybridization for (A, B) Tbx4 and (C, D) Tbx5 was performed on lung at embryonic day (E) 15.5 to identify changes in expression patterns. Tbx4 andTbx5 were expressed by the mesenchyme surrounding the tracheal rings in the normal control embryonic lung tracheas (A, C), whereas Sox9 knockout lungs displayed diffuse expression of (B) Tbx4 and (D) Tbx5 along all the tracheal mesenchyme. (E, F) The Fgf10 expression pattern was lost in the Sox $9^{\Delta / \Delta}$ trachea. (G) Real time RT-qPCR PCR was used to determine whether expression of Tbx4 and Tbx5 mRNA was increased in the mutant mouse trachea versus wild-type mouse trachea $\left({ }^{*} P<0.05\right)$. Fgf10

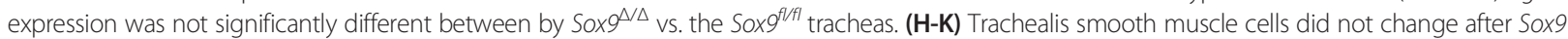
knockout. Staining for Sm-22-a was used to identify the trachealis smooth muscle cells on transverse sections of normal and transgenic mouse tracheas. $(\mathbf{J}, \mathbf{K})$ Even though the absolute area of trachealis smooth muscle cells did not change, the $\mathbf{S m}$-22-a relative area was significantly higher in the $\operatorname{Sox}^{\Delta / \Delta}(\mathbf{J})$ compared with $(\mathbf{K})$ the Sox $9^{\nexists / f}$ trachea, because of the smaller transversal area of the mutant trachea. ${ }^{* *} P=0.015$. Values are mean $\pm S D, n=5$. (L, M). Staining for a-smooth muscle actin (a-SMA) was used to highlight smooth muscle cells. Sox9 deletion did not affect lung smooth muscle cell proliferation or differentiation.

is also a key regulator of embryonic kidney epithelial branching [16]. These contrasting reports on Sox9 regulation of lung epithelial lung branching may be due to the use of different mouse genetic backgrounds by Perl and Chang. Here, we focused on the role of the mesenchymal Sox9-expressing cells, which are localized around the trachea and main bronchi. By using an inducible doxycycline-regulated system, driven by part of the $T b x 4$ gene promoter [8], we were able to delete Sox 9 expression specifically in the mesenchymal compartment of the murine lung. Our collaborator Shi [9] developed the Tbx4-rtTA transgenic mouse line and in the present study, we showed that this mouse line specifically deleted Sox9 in lung mesenchyme (Figure 1D-I) and likewise activated a green fluorescent protein (GFP) reporter gene in lung mesenchyme (Figure 1J). 


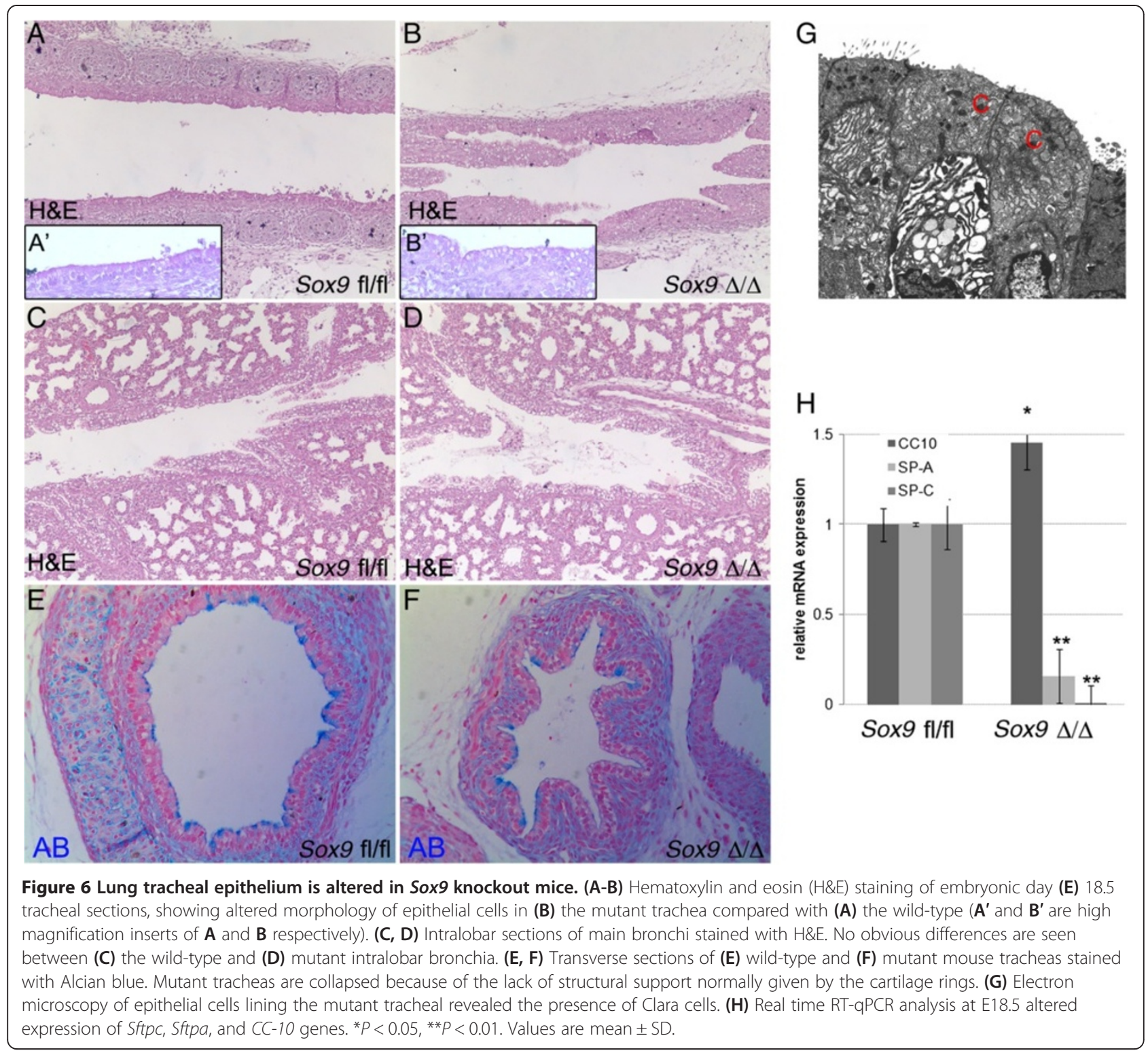

Mice lacking Sox9 did not develop tracheal cartilage rings, were unable to breathe because of tracheal collapse, and died shortly after birth. Transgenic mice appeared normal at birth, but then rapidly showed clear evidence of cyanosis (Figure 2A), and not a single transgenic pup lived more than a few hours after birth (Figure 2B), despite the birth weights of the mutants being similar to those of wild-type mice (Figure 2C). These data suggest that lack of mesenchymal Sox9 in embryonic lung is not compatible with life.

Arora et al. obtained a similar but milder tracheal cartilage phenotype in transgenic mice missing both the $T b \times 4$ and $T b \times 5$ genes; some cartilage trachea rings still developed, but they were smaller and had an abnormal shape [7]. A Tbx4 and Tbx5 combined deletion also resulted in altered lung branching. Data from the same laboratory also suggested that $T b \times 4$ and $T b \times 5$ are upstream regulatory genes of $\operatorname{Sox} 9$ in the embryonic lung mesenchyme [7].

Lung mesenchyme Sox9 knockout mice did not show an obvious phenotype on lung branching; the number of terminal branches of the left lobe of E15.5 lungs was not different between the wild-type and mutant embryos (Figure 3A-C). Moreover, differentiation of distal lung epithelium and mesenchyme was not affected by lack of Sox9 mesenchymal expression at E18.5 (Figure 3D-G). Thus, Sox9 deletion appears to affect only tracheal development.

In our mesenchyme-specific Sox 9 knockout model, $T b \times 4$ and $T b \times 5$ expression was enhanced in the trachea. This indicates the existence of a complex feedback from $T b x 4 / 5$ to Sox 9 and vice versa. Thus, Tbx4/5 gene 

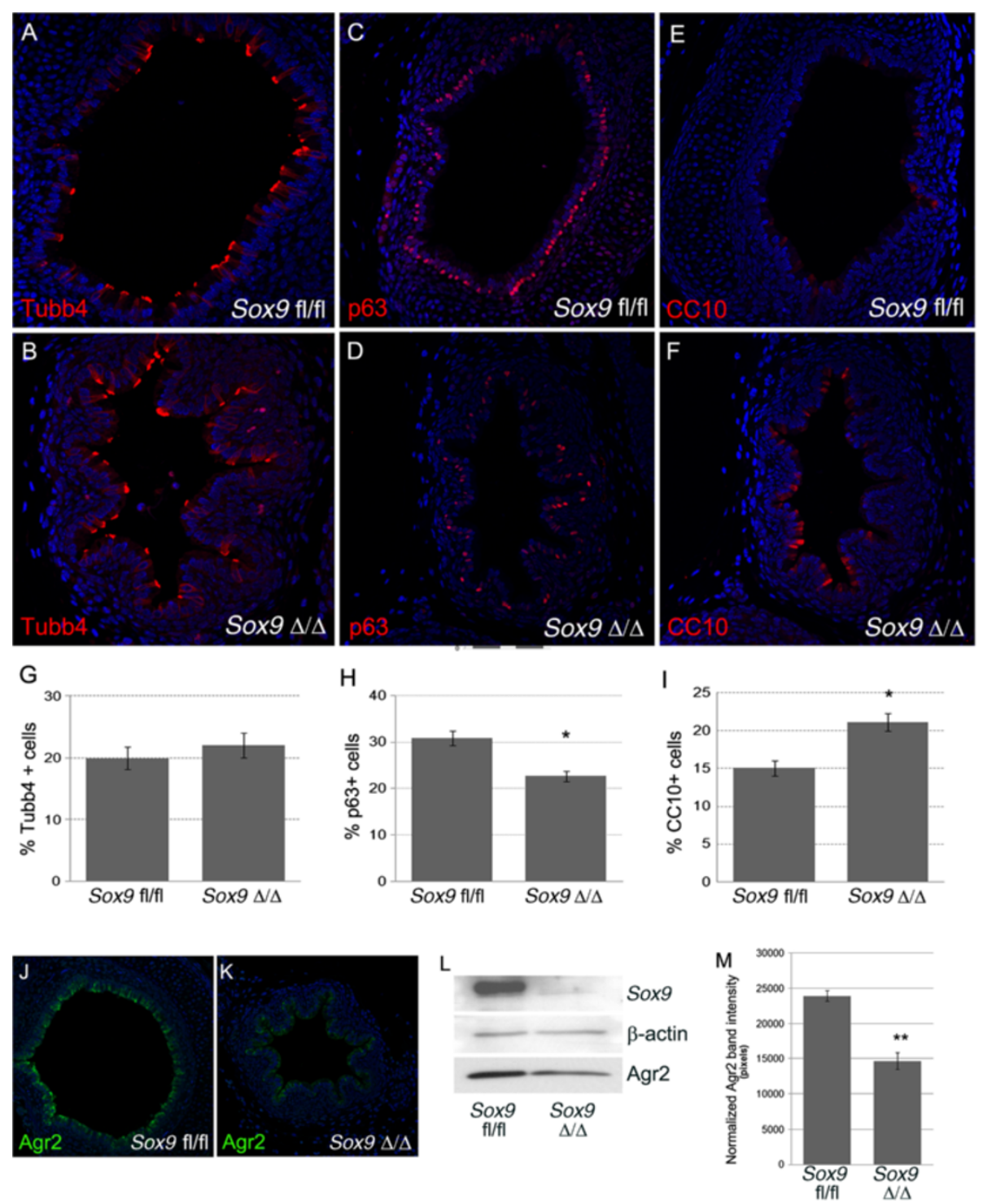

Figure 7 Basal cell numbers are decreased and Clara cell numbers are increased in the mutant mouse trachea. (A-B) Ciliated cell number was not altered in Sox9 knockout mouse tracheas. Staining for $\beta$-tubulin-IV on transverse section of wild-type and mutant mouse trachea was used to determine change in ciliated cell numbers. (G) The number of ciliated cells did not change in the mutant mouse trachea versus the wild-type mouse trachea. (C, D) Staining for P63 was used to confirm that the number of basal cells is decreased in the mutant mouse trachea. (E, F) CC10 marker staining was used to highlight the increase in Clara cell number in Sox9 mutant mouse trachea. (G-I) Cell count statistics. ${ }^{*} P<0.03$. Values are mean $\pm S D, n=4$. (J-M) Sox $9^{\Delta / \Delta}$ tracheas had a reduced number of goblet cells. $(\mathbf{J}, \mathbf{K})$ Immuno-fluorescence staining and (L) western blot for Agr2 protein were used to determine change in goblet cells after Sox9 knockout. Sox9 knockout resulted in reduced production of Agr2 by the tracheal epithelium, suggesting reduced numbers of goblet cells in the Sox $9^{\Delta / \Delta}$ trachea. (M) Intensity analysis of Western blot bands. ${ }^{* *} P<0.05$. Values are mean \pm SD.

expression may have been upregulated, and may thus have partially compensated for the lost Sox 9 expression in our mutant.

Moreover, Sox 9 knockout mice also had an altered Tbx4/5 pattern of expression. In normal lung trachea, the $T b \times 4$ and $T b \times 5$ genes are expressed by the mesenchyme in the intervals between the developing cartilage rings. Thus, the $T b \times 4 / 5$ genes and the Sox9 gene are normally mutually exclusive in the tracheal mesenchyme at E15.5. When the Sox9 gene was knocked out, the $T b \times 4 / 5$ genes were uniformly expressed by all the mesenchyme surrounding the trachea. A similar result was obtained with Fgf10 pattern expression. At E15.5, Fgf10 is normally expressed by the developing cartilage rings in the trachea. Inactivation of Sox9 resulted in the disruption of the normal Fgf10 spatial expression pattern; Fgf10 was now expressed by all the mesenchyme surrounding the trachea. Quantification of the mRNA 


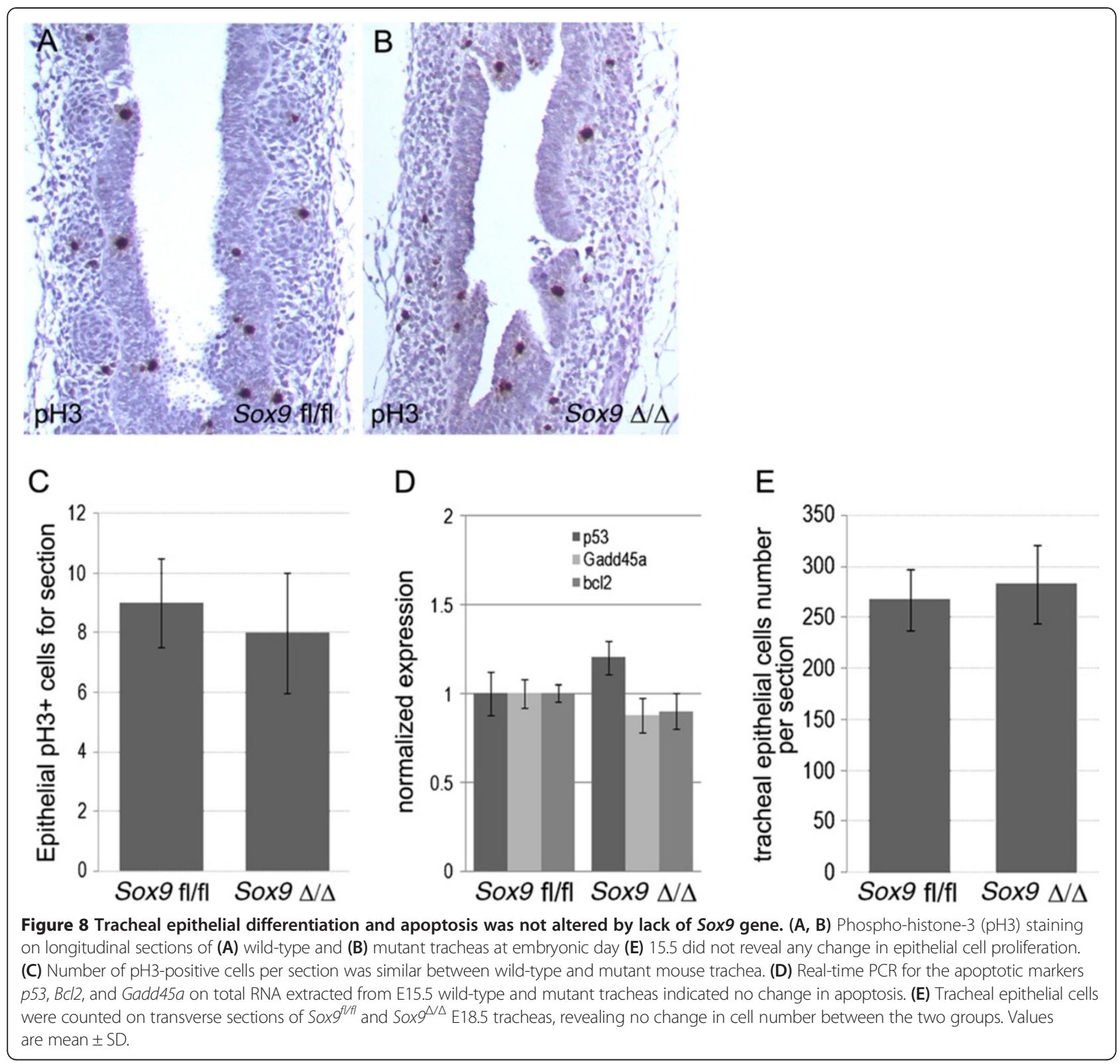

of Fgf10 did show a small trend towards a decrease in mRNA level, but this did not reach statistical significance.

Taken together, these data clearly indicate that the Sox9 gene has a key role in patterning the expression of $T b \times 4 / 5$ and $F g f 10$ in the embryonic trachea. Genes linking Sox9 expression to $T b x 4 / 5$ and Fgf10 gene expression pattern will be the subject of future studies.

The Tbx4-rtTA inducible system allowed us to closely regulate the timing of Sox 9 deletion. We took advantage of this to investigate how the length of time of doxycycline induction relates to the severity of the phenotype. We found that shorter induction resulted in a milder phenotype. If the doxycycline was removed from the food at E11.5, the two most distal cartilage rings formed fully (Figure 4G), but in these mice, the $T b x 4$ gene was expressed by all the lung mesenchyme [7], which implies that all lung mesenchymal cells in these mice have a non-functional Sox9 gene at that stage. There are several possible explanations for these results. It could be that mesenchymal Sox9-positive cells are continuously migrating from the laryngeal region into the tracheal mesenchyme to contribute to cartilage ring formation, or that the shorter doxycycline exposure results in incomplete Sox9 deletion. Another possible explanation is that Tbx4-rtTA is expressed in distal mesenchyme and then proceeds towards the larynx over time. Future studies involving the use of a Sox9-ER-cre mouse line in combination with our Tbx4-rtTA model should be able to 

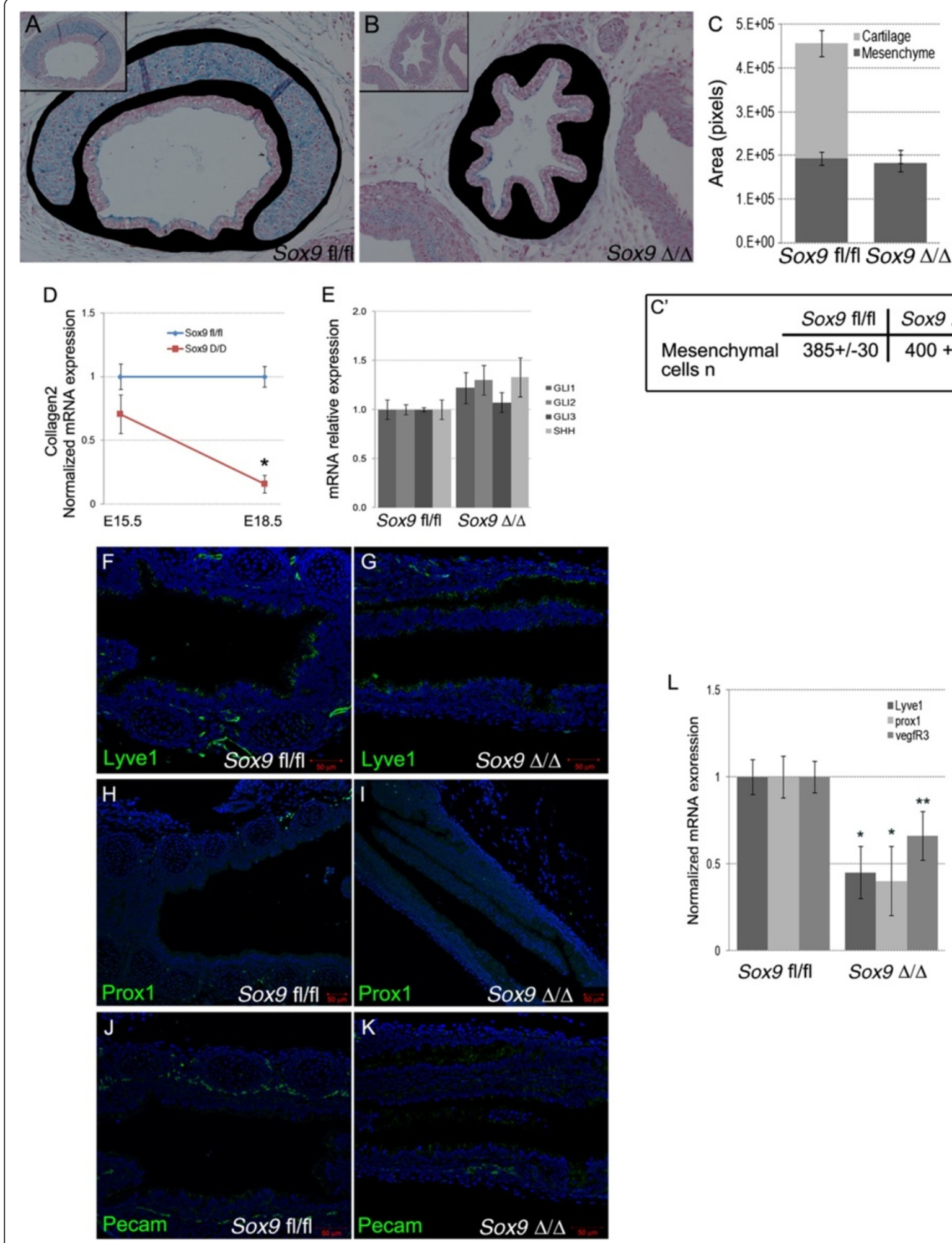

Figure 9 (See legend on next page.) 
(See figure on previous page.)

Figure 9 Lymphatic vascular system was affected by Sox 9 deletion. (A-C) Tracheal mesenchyme area quantification analysis did not reveal changes between the wild-type and mutant tracheas (tracheal mesenchyme was stained in black). (C') The number of mesenchymal cells per section was similar between wild-type and mutant trachea. (D, E) Real time RT-qPCR for Collagen2, Gli1, and Shh on mRNA from wild-type and mutant tracheas at embryonic day (E) 15.5. Col2 expression was decreased in the Sox $9^{\Delta / \Delta}$ trachea starting at E15.5 stage. No statistically significant differences in expression of Shh, Gli1, Gli2, or Gli3 was observed between Sox $9^{f l / f l}$ and Sox $9^{\Delta / \Delta}$. (F-L) The lymphatic vascular system was underdeveloped

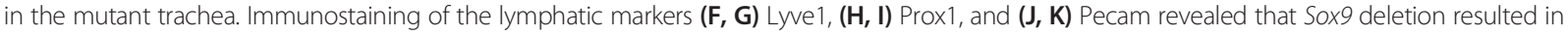
impaired lymphatic vessel formation. (L) Real time RT-qPCR for Prox, Lyve1, and Vegfr3 confirmed that the lymphatic vascular system was not properly developed in the Sox9 knockout tracheas. ${ }^{*} P<0.05,{ }^{*} P=0.08$.

determine which explanation is likely to be the correct one. However, considering that $\operatorname{Sox} 9$ is also expressed by neural crest cells $[17,18]$, which are 'migratory' cells, the hypothesis of Sox9 continuously migrating into the lung mesenchyme seems to be the most plausible, and it will be the first hypothesis to be tested in our future studies. By E14.5 to E15.5, tracheal Tbx4 and Sox9 are mutually exclusively expressed, thus as predicted, knockout of the Sox9 gene atat this later stage (E14-5 to E15-5) did not affect tracheal cartilage development (Figure 4). The ability to regulate the severity of the tracheal phenotype will allow us to tailor timed knockouts to model specific tracheal and lung disease models for future studies.

As pointed out above, mesenchymal-epithelial crosstalk is required for correct development of the embryonic lung. Therefore, anatomical or molecular alterations of one embryonic compartment such as mesenchyme may affect the correct development of the epithelia, and vice versa. We noticed that the tracheal epithelium was altered after mesenchymal Sox9 knockout. Cell count analysis revealed that mutant trachea had a lower number of basal $\mathrm{P} 63+$ cells, and a higher number of Clara $(\mathrm{CC} 10+)$ cells. The number of ciliated cells did not change. An immediate conclusion would be that the absence of cartilage rings around the embryonic trachea caused the tracheal epithelium to behave more similarly to the more distal intralobar bronchiolar epithelium. As the number of basal cells was not altered in the E15.5 Sox $9^{\Delta / \Delta}$ trachea, we suggest that basal cell differentiation is not directly dependent on Sox9 expression but is more likely to depend on cartilage condensation, which normally starts to occur at E15.5. Thus, we speculate that a specific growth factor signal (or signals) may be released by the cartilage rings to promote correct tracheal epithelium differentiation.

$\operatorname{Sox} 9^{\Delta / \Delta}$ trachea had reduced production of Agr2, a protein that identifies goblet cells in the lung. Moreover, transcripts of both surfactant proteins $\mathrm{A}$ and $\mathrm{C}$ were reduced in the mutant trachea, further supporting the role of Sox9 expressing cells in the differentiation of tracheal epithelium.

Despite the altered differentiation, proliferation of tracheal epithelium was not affected by lack of mesenchymal Sox9 expression. No change in $\mathrm{pH} 3$ or epithelial cell number per transversal section was found (Figure 8A-E).
Thus, lack of tracheal rings altered proper differentiation of tracheal epithelium but not its proliferation.

The expression pattern of Fgf10 in $\operatorname{Sox} 9^{\Delta / \Delta}$ embryonic trachea was markedly altered. Even though it was not statistically significant, Fgf10 mRNA level was slightly decreased in the mutant trachea. Volckaert et al. [16] showed that Fgf10 overexpression resulted in an increased number of basal cells and decreased number of Clara cells [19]. Because of the concordance of these and our results, we hypothesize that incorrect expression of Fgf10 may be mostly responsible for the altered differentiation of tracheal epithelium in the $S_{0 x} 9^{\Delta / \Delta}$ trachea. We cannot conclude without any doubt that the epithelial phenotype is directly related to the absence of signals originating from the cartilage rings. An alternative plausible model is that the lack of mechanical support (and thus collapse) of the trachea instructs the epithelium to differentiate onto a different path. Finally, the lack of proper vasculature development (discussion below) in the mutant trachea may also contribute to the altered differentiation of the tracheal epithelium.

$\mathrm{Gli}^{-/-}, \mathrm{Gli}^{+} /-$mutants displayed esophageal atresia with tracheo-esophageal fistula and a severe lung phenotype [20]. Gli2 and Gli3 gene expression in the $\operatorname{Sox} 9^{\Delta / \Delta}$ trachea did not change in our Sox9 knockout model (Figure 9E). Shh and Gli1 are also key regulators of cartilage development [21], but their expression was not altered in our $\operatorname{Sox} 9^{\Delta / \Delta}$ embryonic trachea (Figure 9E). Expression of Collagen2, a key regulator of cartilage differentiation, was reduced after Sox9 deletion, supporting the model in which $\operatorname{Sox} 9$ acts upstream of Collagen 2 to control tracheal cartilage development.

Despite the somewhat misleading visual appearance, the numbers of mesenchymal cells and the area covered by them were not different between the $S_{0 x} 9^{f l / f l}$ and $S_{0 x} 9^{\Delta / \Delta}$ trachea (Figure 9A-C,C'). Thus, the apparent difference in size observed between the wild-type and mutant tracheas is caused by the lack of the cartilage rings in the $\operatorname{Sox}^{4 / \Delta}$ trachea. Pecam is broadly expressed by vascular structures [22,23]. and its expression in the distal lung mesenchyme was assayed to investigate any alterations of distal mesenchyme lung differentiation. As we observed for the distal epithelium, the distal lung mesenchyme appeared normal in the mutant trachea. We concluded that lack of Sox9 in the lung mesenchyme did not affect the differentiation or development of the vasculature in the distal lung mesenchyme. 
The lymphatic vascular system serves to transport tissue fluid, extravasated plasma proteins, and cells back to the circulation [24,25]. Prox1 and Lyve1 are key regulators and markers of lymphatic vessel development. The lymphatic system was significantly underdeveloped in the mutant Sox9 tracheas; immunostaining of Prox1, Lyve1, and Pecam, and mRNA level of Prox1, Lyve1, and Vegfr3 were reduced in the $\operatorname{Sox}^{\Delta / \Delta}$ tracheas. Thus, lack of tracheal cartilage affects appropriate tracheal lymphatic vessel development. It is also possible that an early Sox9-expressing mesenchymal cell population exists, and that this differentiates into lymphatic vessel cells. As Pecam is expressed by both blood and lymphatic vascular vessels, we speculate that mesenchymal Sox9 expression has a broad role in the generalized vascularization of the trachea. The mechanism linking the lack of cartilage rings to the altered tracheal vascularization is under further investigation. We focused on epidermal growth factor, as it is known to be involved in lymphatic vessel modeling in the skin [26], and found it to be downregulated in the $\operatorname{Sox} 9^{\Delta / \Delta}$ trachea (data not shown).

\section{Conclusions}

In conclusion, we have uncovered a key fundamental role of the mesenchymal Sox9-expressing cells in the development of the tracheal cartilage rings, which in turn induce proper tracheal epithelium differentiation and lymphatic system development (Figure 10). Thus, besides having a mechanical function, tracheal cartilage also appears to be a local signaling structure in the embryonic lung. Some pieces of this puzzle are still missing, and are currently under investigation. Nevertheless, we have identified an important mechanism by which mesenchymal Sox9expressing cells instruct tracheal cartilage, epithelial, and lymphatic differentiation in a phenotype that already appears to model and may perhaps explain mechanistically some extreme cases of primary tracheomalacia in human new born infants.

The appropriate temporospatial expression of Sox9 in lung mesenchyme is necessary for correct tracheal cartilage formation, lymphatic vasculature system development, and epithelial differentiation. Our study showed that a lack of tracheal cartilage rings resulted in increase in Clara cells and decrease in basal and goblet cells in the tracheal epithelium. We also uncovered a novel mechanism of lung epithelium differentiation; tracheal cartilage rings instruct the tracheal epithelium to differentiate properly during embryonic development. Thus, besides having a mechanical function, tracheal cartilage also appears to be a local signaling structure in the embryonic lung.

\section{Methods}

\section{Ethics statement}

We strictly followed the National Institute of Health guidelines for animal care and safety. The animal

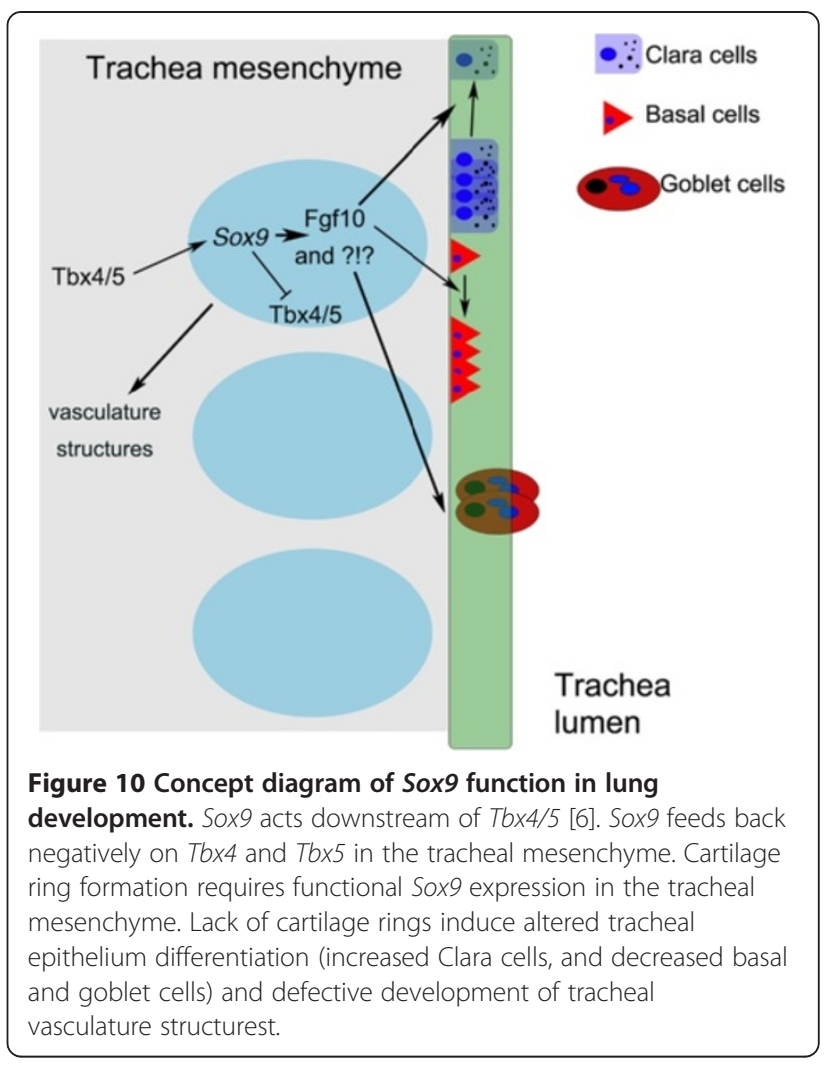

experimental protocols were approved by the Animal Care and Use Committee at the Children's Hospital Los Angeles (CHLA), where the vivarium is under weekly surveillance by an external chief veterinarian to check animal health. Our animal facility is approved by the AAALAC (Association for Assessment and Accreditation of Laboratory Animal Care) and USDA (The United States Department of Agriculture).

\section{Mice}

Sox $9^{f l / f l}$ mice were bought from Jackson Laboratories (Sacramento, CA). Tbx4-rtTA mice were kindly provided by W Shi (CHLA) [9]. The plasmid bearing the lung-specific enhancer of $T b x 4$ was kindly provided by D B Menke (University of Georgia) [8]. Tet-On-Cre mice were bought from Jackson Laboratories. Mt/mG mice (stock number 007576) were bought from Jackson Laboratories. Tbx4-rtTA/Tet-on-cre mice were generated and crossed with female Sox $9^{f l f l}$. Timed pregnant mice were fed with doxycycline chow (rodent diet with $0.0625 \%$ doxycycline; Harlan Teklad TD01306) from E7.5 stage, unless specified.

\section{Alcian blue staining}

Alcian blue staining was performed in accordance with standard protocols. Lungs and trachea were dissected out at different stages of life, and fixed in 95\% ethanol 
for 1 to 2 days, stained in Alcian blue solution for 1 day, washed with $95 \%$ ethanol for 1 to 2 days, and finally clarified in $1 \%$ potassium hydroxide solution for 2 to 5 hours [5]. For Alcian blue staining on tissue sections, 5 $\mu \mathrm{m}$ thick paraffin wax-embedded sections were rehydrated, stained with $1 \%$ Alcian blue in 3\% acetic acid for 30 minutes, washed in distilled water, and counterstained with Nuclear Fast Red.

\section{Whole-mount in situ hybridization}

E15.5 lungs were isolated from embryos and fixed for 2 hours in $4 \%$ paraformaldehyde in phosphate-buffered saline (PBS). The samples were washed twice in PBS for 5 minutes, transferred to $70 \%$ ethanol overnight, and stored in $100 \%$ ethanol until needed. Whole-mount in situ hybridization was performed as previously described, with riboprobes transcribed from murine plasmid DNA templates [5].

\section{Immunohistochemistry and histology}

Immunohistochemistry and immunofluorescence were performed on $5 \mu \mathrm{m}$ thick paraffin wax-embedded sections, as described previously. Primary antibodies used were anti-Sm22- $\alpha$, P63, Sox9, CC10 (Santa Cruz Biotechnology), anti-Tubb4 (BioGenex), (Developmental Studies Hybridoma Bank antibody 8.1.1), anti-Agr2 (Abgent), anti-phospho-H3, $\beta$-catenin (Cell Signaling Technology, Danvers, MA), $\alpha$-SMA (Sigma-Aldrich), anti-PECAM (LifeSpan Bioscences Inc.), anti-Prox1, and Lyve1 (both Angiobio). The Alexa Fluor 488, 555, and 647 secondary antibodies were from Invitrogen.

For histological evaluation, embryos were removed from the uterine horns, and dissected free from the decidua, then the lungs were removed and fixed in $4 \%$ formalin at room temperature. After dehydration in ethanol, embryos were embedded in paraffin wax, sectioned at $5 \mu \mathrm{m}$ thickness and stained with hematoxylin and eosin or Alcian blue.

\section{CDNA reverse transcription and real-time reverse transcription PCR}

Total RNA was extracted from mouse tracheas using Trizol reagent, then 500 to $1,000 \mathrm{ng}$ of RNA was reverse-transcribed using the IscriptcDNA synthesis kit (Bio-Rad, Hercules, CA, USA). RNA was extracted from a pool of three tracheas to get sufficient goodquality RNA for expression analysis. Real-time quantitative PCR was performed in a Lightcycler II (Roche Applied Science) using gene-specific primers (Operon, Huntsville, AL, USA) and fluorescence probes (Roche Applied Science).

\section{Western blot}

Embryos were dissected in cold PBS, tracheas were separated from the esophagus and lysed with RIPA buffer
$(1 \times$ Tris-buffered saline, $1 \%$ Nonidet P-40, $0.5 \%$ sodium deoxycholate, $0.1 \%$ SDS, $0.004 \%$ sodium azide; Sigma-Aldrich, St. Louis, MO, USA), supplied with phosphatase and protease inhibitors (phenylmethylsulfonyl fluoride (PMSF) solution, sodium orthovanadate solution, and protease inhibitor cocktail solution; Sigma-Aldrich). Three tracheas from each experimental group (wild-type and control) were combined together to achieve a sufficient amount of protein lysate. $\beta$-actin protein amount was used as loading control and measured in each western blot experiment.

\section{Cell count and cellular area analysis}

A Leica confocal microscope (Leica) was used to capture fluorescent images. For epithelial and mesenchymal cells counts, (Figure 8C, Figure 9C), $4 \mu \mathrm{m}$ thick tracheal transversal sections were used; nuclei were highlighted with DAPI or hematoxylin, and counted with ImageJ software.

For mesenchymal area analysis, the mesenchyme area on transversal sections of tracheas was highlighted, and pixels were counted using Photoshop software.

\section{Statistical analysis}

The student's t-test was used to perform statistical analysis. Every experiment was repeated at least three times unless indicated.

\section{Additional files}

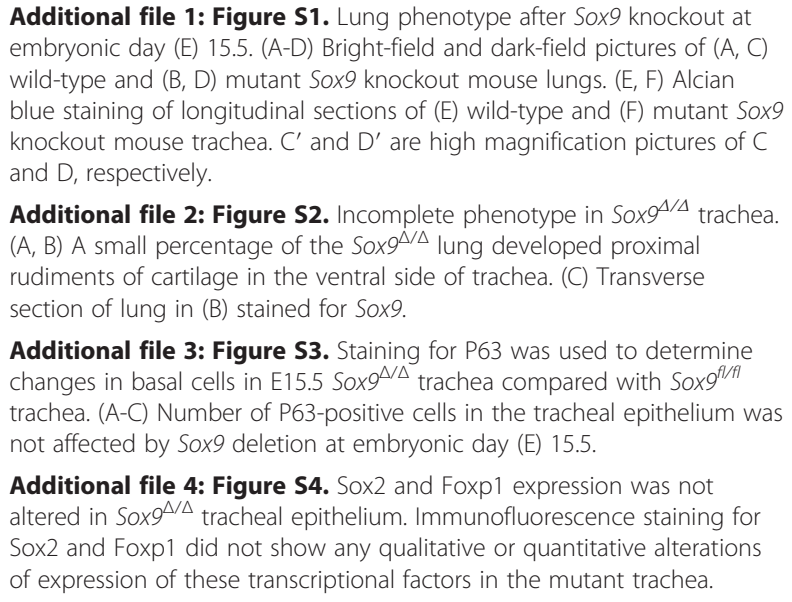

Additional file 3: Figure S3. Staining for P63 was used to determine changes in basal cells in E15.5 Sox $9^{\Delta / \Delta}$ trachea compared with Sox $9^{f / / f l}$ trachea. (A-C) Number of P63-positive cells in the tracheal epithelium was not affected by Sox9 deletion at embryonic day (E) 15.5

Additional file 4: Figure S4. Sox2 and Foxp1 expression was not altered in $\operatorname{Sox}^{\Delta / \Delta}$ tracheal epithelium. Immunofluorescence staining for Sox 2 and Foxp1 did not show any qualitative or quantitative alterations of expression of these transcriptional factors in the mutant trachea.

\section{Competing interests}

The authors declare that they have no competing interests. 


\section{Acknowledgments}

We thank Dr. Mark Frey and Sue Buckley for critical editing of the manuscript.

This work was funded by The Pasadena Guild of Children's Hospital Los Angeles Endowment, by The Garland Foundation, The Webb Foundation and by a California Institute of Regenerative Medicine Training Grant to DW

\section{Author details}

${ }^{1}$ Developmental Biology and Regenerative Medicine Program, Saban Research Institute, Children's Hospital Los Angeles, Keck School of Medicine and Ostrow School of Dentistry, University of Southern California, 4661 Sunset Boulevard, Los Angeles, CA 90027, USA. ²Department of Genetics, University of Georgia, 120 East Green Street, Athens, GA 30602, USA ${ }^{3}$ Department of Biosciences, Occidental College, 1600 Campus Road, Los Angeles, CA 90041, USA

Received: 5 November 2013 Accepted: 22 November 2013 Published: 25 November 2013

\section{References}

1. Whitsett JA, Wert SE, Trapnell BC: Genetic disorders influencing lung formation and function at birth. Hum Mol Genet 2004, 13:207-215.

2. Goo HW: Free-breathing cine $\mathrm{CT}$ for the diagnosis of tracheomalacia in young children. Pediatr Radiol 2013, 42:922-928.

3. Kandaswamy $C$, et al: Severe tracheomalacia in the ICU: identification of diagnostic criteria and risk factor analysis from a case control study. Respir Care 2013, 58:340-347.

4. Goldring MB, Tsuchimochi K, ljiri K: The control of chondrogenesis. J Cell Biochem 2006, 97:33-44.

5. Sala FG, et al: FGF10 controls the patterning of the tracheal cartilage rings via Shh. Development 2011, 138:273-282.

6. Elluru RG, Thompson F, Reece A: Fibroblast growth factor 18 gives growth and directional cues to airway cartilage. Laryngoscope 2009, 119:1153-1165.

7. Arora R, Metzger RJ, Papaioannou VE: Multiple roles and interactions of Tbx4 and Tbx5 in development of the respiratory system. PLoS Genet 2012, 8:e1002866.

8. Menke DB, Guenther C, Kingsley DM: Dual hindlimb control elements in the Tbx4 gene and region-specific control of bone size in vertebrate limbs. Development 2008, 135:2543-2553.

9. Zhang $W$, et al: Spatial-temporal targeting of lung-specific mesenchyme by a Tbx4 enhancer. BMC Biol 2013, 11:111.

10. Perl AK, et al: Normal lung development and function after Sox 9 inactivation in the respiratory epithelium. Genesis 2005, 41:23-32.

11. Chang DR, et al: Lung epithelial branching program antagonizes alveolar differentiation. Proc Natl Acad Sci U S A 2013, 110:18042-18051.

12. Mishima $K$, et al: Prox 1 induces lymphatic endothelial differentiation via integrin alpha9 and other signaling cascades. Mol Biol Cell 2007, 18:1421-1429.

13. Kaltezioti $V$, et al: Prox1 regulates the notch1-mediated inhibition of neurogenesis. PLOS Biol 2010, 8:e1000565.

14. Rackley $C R$, Stripp BR: Building and maintaining the epithelium of the lung. J Clin Invest 2012, 122:2724-2730.

15. Warburton D, et al: Lung organogenesis. Curr Top Dev Biol 2010, 90:73-158.

16. Reginensi A, et al: SOX9 controls epithelial branching by activating RET effector genes during kidney development. Hum Mol Genet 2011, 20:1143-1153

17. Mori-Akiyama $Y$, et al: Sox9 is required for determination of the chondrogenic cell lineage in the cranial neural crest. Proc Natl Acad Sci U S A 2003, 100:9360-9365.

18. Sahar DE, Longaker MT, Quarto N: Sox9 neural crest determinant gene controls patterning and closure of the posterior frontal cranial suture. Dev Biol 2005, 280:344-361.

19. Volckaert T, et al: Localized Fgf10 expression is not required for lung branching morphogenesis but prevents differentiation of epithelial progenitors. Development 2013, 140:3731-3742.

20. Motoyama J, et al: Essential function of Gli2 and Gli3 in the formation of lung, trachea and oesophagus. Nat Genet 1998, 20:54-57.

21. Park J, et al: Regulation of Sox 9 by Sonic Hedgehog (Shh) is essential for patterning and formation of tracheal cartilage. Dev Dyn 2010, 239:514-526.

22. Jackson DE: The unfolding tale of PECAM-1. FEBS Lett 2003, 540:7-14
23. Liersch $\mathrm{R}$, et al: Induction of lymphatic endothelial cell differentiation in embryoid bodies. Blood 2006, 107:1214-1216

24. Karkkainen MJ, Alitalo K: Lymphatic endothelial regulation, lymphoedema, and lymph node metastasis. Semin Cell Dev Biol 2002, 13:9-18.

25. Karkkainen MJ, Makinen T, Alitalo K: Lymphatic endothelium: a new frontier of metastasis research. Nat Cell Biol 2002, 4:E2-E5.

26. Marino D, et al: Activation of the epidermal growth factor receptor promotes lymphangiogenesis in the skin. J Dermatol Sci 2013, 71:184-194.

doi:10.1186/1741-7007-11-117

Cite this article as: Turcatel et al: Lung mesenchymal expression of Sox 9 plays a critical role in tracheal development. BMC Biology 2013 11:117.

\section{Submit your next manuscript to BioMed Central and take full advantage of:}

- Convenient online submission

- Thorough peer review

- No space constraints or color figure charges

- Immediate publication on acceptance

- Inclusion in PubMed, CAS, Scopus and Google Scholar

- Research which is freely available for redistribution 